\title{
Submm VLBI toward Shadow Image of Super Massive Black Hole
}

\author{
Inoue, $\mathbf{M}^{1}$ \\ ${ }^{1} A S I A A$ \\ P.O. Box 23-141, Taipei, 10617 Taiwan, R.O.C. \\ E-mail: inouelasiaa.sinica.edu.tw
}

Blundell, R. ${ }^{2}$, Brisken, W. ${ }^{3}$, Chen, M.T. ${ }^{1}$, Doeleman, S. ${ }^{4}$, Fish, V. ${ }^{4}$, Ho, P. ${ }^{1,2}$, Moran, $\mathrm{J.}^{2}$, Napier, $\mathrm{P}^{3}$, and the Greenland Telescope (GLT) Team

${ }^{2} C f A$

60 Garden Street, Cambridge, MA 02138, USA

${ }^{3} N R A O$

Array Operation Center, P.O. Box O, 1003 Lopezville Road, Socorro, NM 8701-0387, USA

and ${ }^{4}$ MIT Haystack Observatory

Off Route 40, Westford, MA 01886-1299, USA

\begin{abstract}
We are deploying a new submm VLBI station to get shadow images of Super Massive Black Hole (SMBH). Submm VLBI is thought to be the only way to get the direct image of SMBH by its shadow, thanks to the superb angular resolution and high transparency against dense plasma around SMBH. At the Summit Station on Greenland, we have started monitoring the opacity at submm region. The Summit Station subtends long baselines with ALMA in Chile and SMA in Hawaii. In parallel, we started retrofitting the ALMA-NA prototype telescope (renamed as Greenland Telescope: GLT) for the cold weather condition.
\end{abstract}

Resolving the Sky - Radio Interferometry: Past, Present and Future

Manchester, UK

April 17-20, 2012

Presenter: Inoue, M. 


\section{Introduction}

An experiment in the sub-millimeter (submm) Very Long Baseline Interferometry (VLBI) has shown the capability for direct imaging of Sumer Massive Black Hole (SMBH) [1]. The spatial resolution reaches the expected size of several tens of micro arcsec ( $\mu$ as) for some of SMBHs. Submm observations also allow us to see the immediate vicinity of SMBH, penetrating into the dense plasma around it. Supported by imaging simulations [e.g., 2, 3, 4] and good mass estimations of SMBHs $[5,6]$, we are now confident to be able to observe and resolve the shadow image of SMBHs: Sgr A* and M87 being the best targets based on their apparent angular sizes.

The image of SMBH can be seen as a shadow against bright accreting materials, and the enlarged shadow size by a lensing effect of the strong gravity is expected to be about 5 times of the Schwarzschild radius for non rotating black hole. The shadow size depends on the black hole spin [2]. This means we could measure directly the key parameters of black hole, i.e., the mass and spin of SMBH by the imaging with submm VLBI, in addition to observe the other physical conditions under strong gravity field such as the energy transfer in the accretion flows and the launching mechanism of relativistic jets.

However, the number of the submm VLBI telescopes is very limited, and the distribution of baselines, or the $u v$ coverage, is not well arranged. Under such circumstances, development of new submm VLBI sites is a key to promote the SMBH science.

Academia Sinica Institute of Astronomy and Astrophysics (ASIAA) has awarded a new submm telescope with CfA and started a site development for the telescope to establish a new submm VLBI station. We will describe below the science target and the siting situation.

\section{Science Target}

As the Schwarzschild radius $\mathrm{R}_{\text {sch }}$ is proportional to the SMBH mass, the apparent size of the shadow depends on the mass and distance. Table 1 lists these parameters for some SMBHs which have larger apparent size in terms of $\mathrm{R}_{\text {sch. }}$.

Table 1. Apparent size of SMBH shadow

\begin{tabular}{cccc}
\hline Target & $\begin{array}{c}\text { Apparent size } \\
\left(5 \mathrm{R}_{\text {sch }}\right)[\mu \mathrm{as}]\end{array}$ & $\begin{array}{c}\text { Mass } \\
{\left[10^{8} \mathrm{M}_{\text {sol }}\right]}\end{array}$ & $\begin{array}{c}\text { Distance } \\
{[\mathrm{Mpc}]}\end{array}$ \\
\hline Sgr A* & 52 & 0.04 & 0.008 \\
M 87 & 40 & 64 & 16 \\
NGC 4594 (M 104) & 10 & 10 & 10 \\
NGC 4374 (M 84) & 9.7 & 17 & 18 \\
IC 1459 & 9.6 & 26 & 27 \\
Cen A & 1.4 & 0.55 & 4.2 \\
\hline
\end{tabular}

The list suggests that we have now sufficient angular resolution to resolve the black hole shadow for Sgr A* and M87 with ground-based submm VLBI. In terms of the apparent size of the black hole shadow, Sgr A* would be the best target. However, it becomes clear that Sgr A* 
shows rapid time variations within a day even at $\mathrm{mm}$ and submm wavelengths $[7,8]$. This is due to the small mass of the black hole. The rapid variations make it difficult to generate synthesized images of Sgr A*. On the other hand, the mass and size of the SMBH of M87 are $10^{3}$ times larger than that of Sgr $\mathrm{A}^{*}$, and much longer time scale is expected for M87. The SMBH mass of M87 indicates a dynamical timescale of about 5 hours, and a typical orbital timescale at the Innermost Stable Circular Orbit (ISCO) is 2-18 days, depending on the SMBH spin. Therefore, unlike Sgr A*, the structure of M87 may not change during an entire day for the image synthesis. In this point, M87 is better candidate for the VLBI imaging synthesis technique by the Earth rotation. It would be possible to produce a sequence of images of dynamical events in M87, occurring over many days. M87 is thus a promising target to image the black hole shadow and bright accreting materials rotating around it.

Further, as shown in Figure 1, the tracing back of the jet ridgeline is one of the crucial tests to identify the launching point of the jet and constrains the formation mechanism. If the parabolic stream line of the jet extends towards the innermost region, i.e., up to $2 \mathrm{R}_{\text {sch }}$, the jet must originate within the ISCO of the accretion disk for the spinning SMBH and/or the spinning SMBH itself. Each case requires that the SMBH is rotating, which represents a specific solution in the Einstein field equations of general relativity governing a stationary axisymmetric gravitational field [9]. Submm VLBI could make observations of the ridge line to reveal the launching point of the relativistic jet [10].

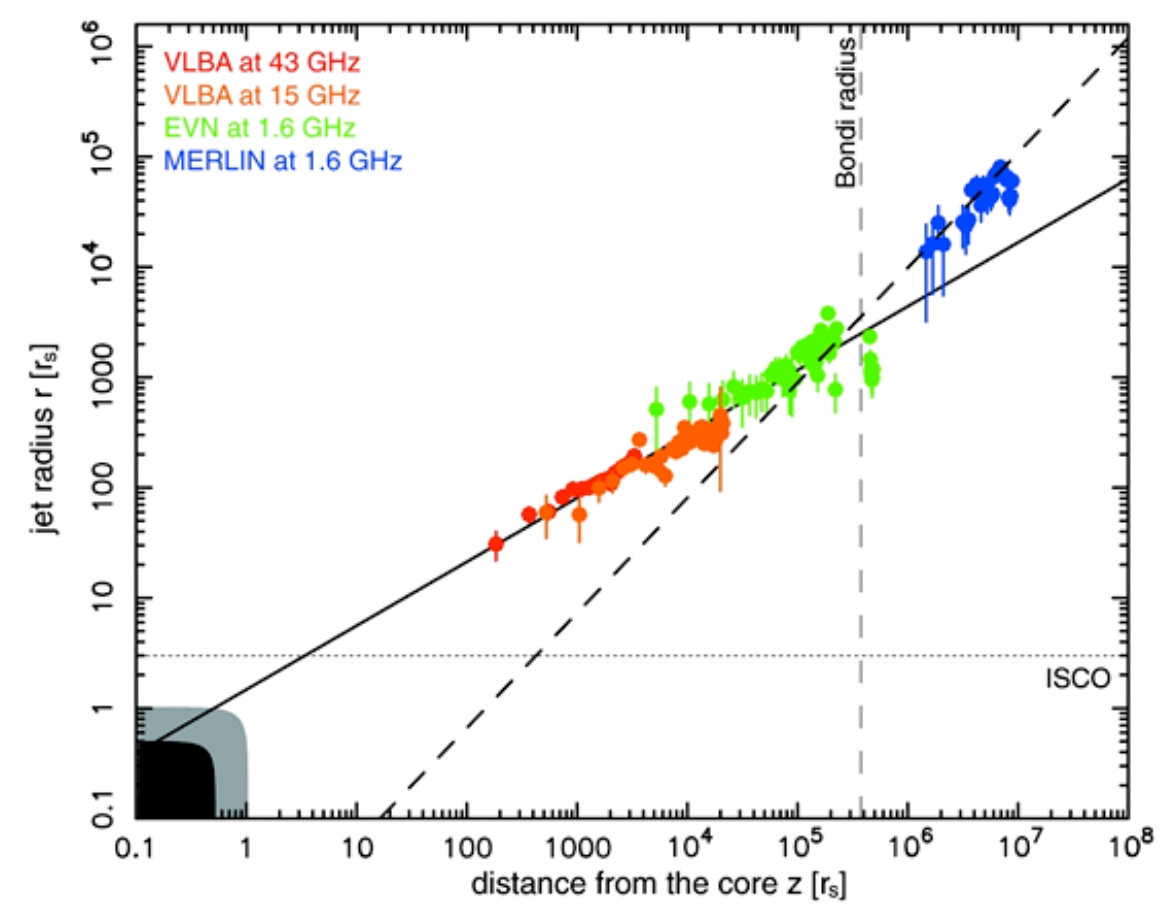

Figure 1. Distribution of the radius of the jet as a function of the deprojected distance $r_{s}$ from the core. Images were obtained by previous VLBA measurements at $43 \mathrm{GHz}$ (red circles) and at $15 \mathrm{GHz}$ (orange circles), EVN measurements at $1.6 \mathrm{GHz}$ (green circles), and MERLIN measurements at $1.6 \mathrm{GHz}$ (blue circles). The jet is described by two different shapes. The solid line indicates a parabolic structure with a power-law index $\alpha \sim 1.7$, while the dashed line 
indicates a conical structure with $\alpha \sim 1.0$. HST-1 is located around $5 \times 10^{5} r_{s}$, near the Bondi radius. The black area near the core shows the size of the minor axis of the event horizon of the spinning black hole with maximum spin. The gray area indicates the size of the major axis of the event horizon of the spinning black hole with maximum spin, which also corresponds to the size of the event horizon of the Schwarzschild black hole. The dotted line indicates the size of the ISCO of the accretion disk for the Schwarzschild black hole [10].

\section{Site Selection and Testing}

To image the vicinity of black hole, observations are planned at $230 \mathrm{GHz}, 350 \mathrm{GHz}$, and even higher frequencies. Atmospheric transmission in these bands strongly depends on the absorption by the column density of water molecules above the site, and it is essential that the precipitable water vapor (PWV) content is less than $1 \mathrm{~mm}$. We thus surveyed a telescope site with dry, cold weather, at a high altitude location, similar to Mauna Kea or the Atacama Desert. Another key point is that the new telescope must be placed where it has common visibilities with the other available submm telescopes, especially those with large aperture sizes to gain high sensitivity, such as the ALMA in Northern Chile and/or the extended SMA (e-SMA) in Hawaii, U.S.A. Furthermore, to get highest angular resolution ever made, the telescopes should be separated as far as possible.

We examined the satellite-based PWV measured by NASA Aqua and Terra/MODIS, and found that Tibet, Alaska, and Greenland show PWV $<3 \mathrm{~mm}$ throughout year. Taking into account the common visibilities with the powerful telescopes of ALMA and e-SMA, Greenland was selected to observe the shadow image of the SMBH in M87, although Sgr A* cannot be observed by its low declination.

Greenland is covered with snow and ice, and the summit of the ice cap is more than 3,000 $\mathrm{m}$ high. Near the summit, polar research facilities are operated with some infrastructures with a runway for air cargos, e.g., C-130 Hercules, supported by NSF, USA. In August 2011, we deployed a tipping radiometer at $225 \mathrm{GHz}$ to monitor the opacity at the Summit Station. Figure 2 shows the monitoring data of opacity tau with temperature. The preliminary conclusions so far are (1) Opacity tau $<0.04$ for $50 \%$ of time (see red curve in Figure 2), (2) Better than SMA site in Hawaii, (3) Comparable condition to ALMA and the South Pole sites, and then (4) Excellent site for both VLBI and single-dish observations.

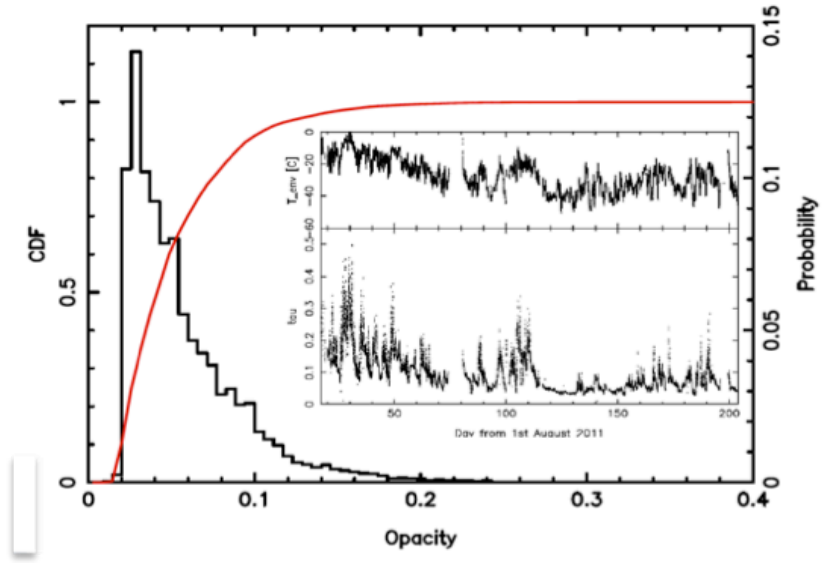

Figure 2. Cumulative Distribution Function (CFD, red curve) and histogram of opacity, and inserted are monitoring data of temperature and opacity. 


\section{Submm VLBI Network}

As seen in Figure 3, the baselines between Greenland, Hawaii, and Atacama in Chile form a big triangle for M87, extending 9,000 km long, which achieves the angular resolution of 20 $\mu$ as at $350 \mathrm{GHz}$. At $230 \mathrm{GHz}$, other submm telescopes like IRAM 30-m (Pico Veleta, Spain), PdBI (France), SMT (Arizona, USA), LMT (Mexico), CARMA (California, USA), etc., will provide a good $u v$ coverage to make plausible quality images of M87. The Summit Station on Greenland has good baselines between both European and American submm telescopes. The North-South baselines produced by the Summit Station and other telescopes provide good opportunity to investigate the jet structure of M87 (see Figure 1).

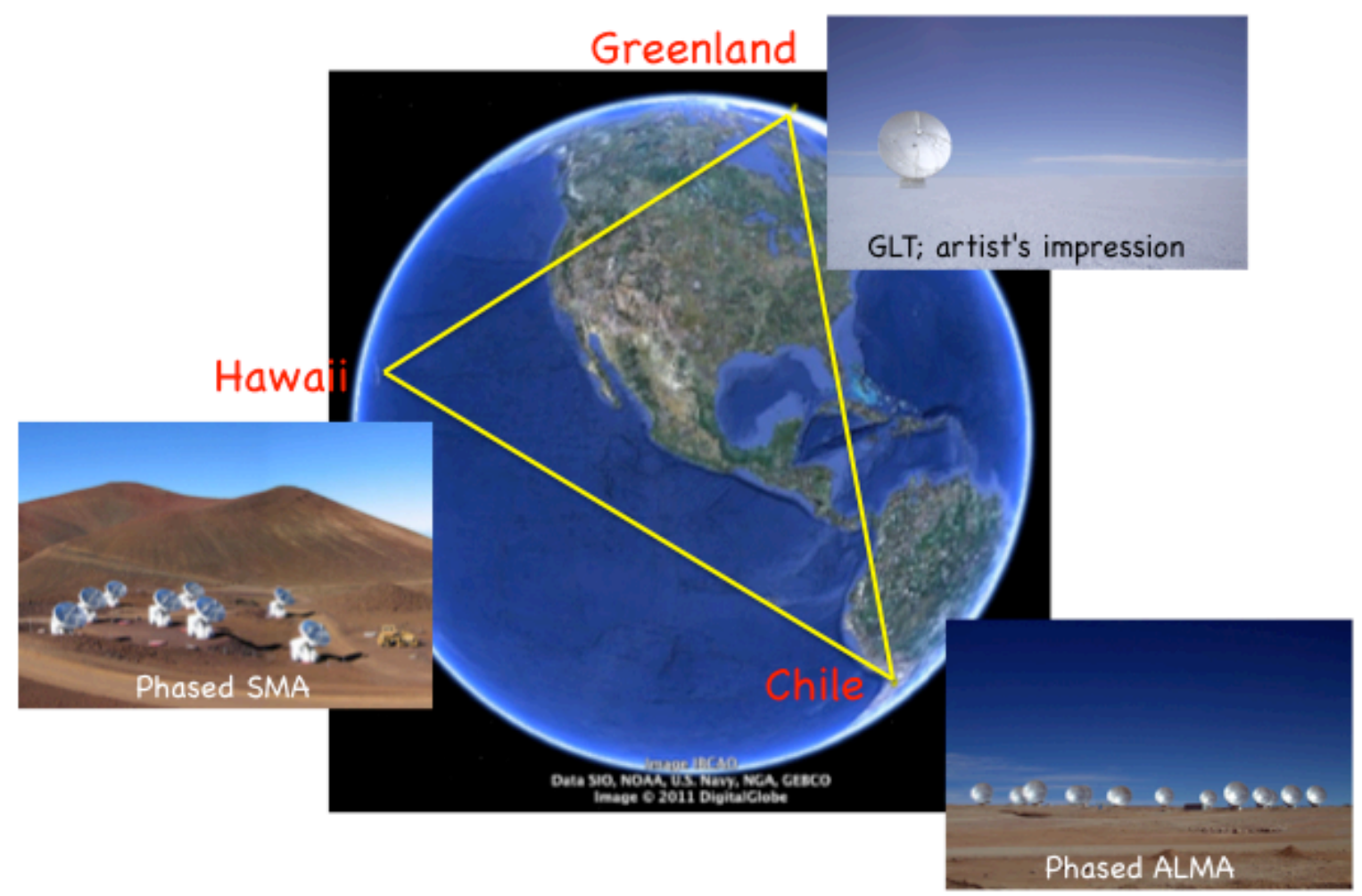

Figure 3. A big triangle formed by the Summit Station on Greenland, SMA in Hawaii, and ALMA in Chile, seen from the direction of M87. This network provides a 20- $\mu$ as resolution at $350 \mathrm{GHz}$.

ASIAA is the only one Institute which has close relation to both SMA and ALMA. The project for phase-up ALMA is in progress with international collaboration, and the phased "SMA, JCMT, and CSO" is already at work by the Haystack Observatory and SAO people. This triangle is then expected to provide high sensitivity and high angular resolution at $350 \mathrm{GHz}$ and even higher frequencies. At $230 \mathrm{GHz}$, many other submm telescopes will collaborate with this big triangle to form a good submm VLBI network. 


\section{Retrofitting Telescope}

Since April 2011, the ALMA-North America prototype telescope has been re-tested and studied to retrofit for the cold environment on Greenland. After retrofitting, the telescope, renamed Greenland Telescope (GLT), will be shipped to Greenland in 2014. In parallel, construction works for the foundation and infrastructures have started, collaborating with a company who operates the summit facilities.

For the submm VLBI receivers, 230, 350, and 650-GHz receivers are planned. The GLT is also expected to be a forerunner of CCAT in science and as a test bench of receiver developments as a single dish telescope. Heterodyne multi feed receivers and multi-pixel bolometers up to $1.5 \mathrm{THz}$ will be installed for the single-dish observations.

\section{Summary}

Direct imaging of SMBH has been anticipated by recent development of submm VLBI. New sites of the submm VLBI network could provide good image quality, and a good site has been identified at the Summit Station of the ice cap on Greenland. ASIAA has been monitoring the sky condition at the Summit Station, and the preliminary monitoring shows satisfactory opacity condition at submm region. The ALMA-NA prototype telescope was awarded to ASIAA/CfA. Retrofitting the telescope, now called GLT, is under way for the Greenland Summit Station. The new submm VLBI network by the big triangle with GLT, ALMA, and SMA, and other submm telescopes will be a powerful tool to imaging the SMBH shadow.

\section{References}

[1] S. Doeleman et al. 2008, Nature, 455, 78.

[2] J.-P. Luminet 1979, $A \& A p, 75,228$.

[3] H. Falcke, F. Melia, \& E. Agol 2000, ApJ, 528, L13.

[4] J. Dexter et al. 2012, MNRAS, 421, 1517.

[5] A.M. Ghez et al. 2008, ApJ, 689, 1044.

[6] K. Gebhardt et al. 2011, ApJ, 729, 119.

[7] A. Miyazaki et al. 2004, ApJ, 611, L97.

[8] V. Fish et al. 2011, ApJ, 727, L36.

[9] R.P. Kerr 1963, Phys. Rev. Lett., 11, 237.

[10]K. Asada \& M. Nakamura 2012, ApJ, 745, L28. 